\title{
Necrotizing Panniculitis as an Uncommon Manifestation of Acute Pancreatitis
}

\author{
Cosimo Marcello Bruno ${ }^{1,2}$, Gabriele Sebastiano Pricoco ${ }^{1}$, Salvatore Bellinvia ${ }^{1}$, Maria Domenica Amaradio ${ }^{1}$, \\ Damiano Cantone ${ }^{1}$, Riccardo Polosa ${ }^{1,2}$ \\ ${ }^{1}$ Unit of Internal and Emergency Medicine, G. Rodolico Hospital, A.O.U. "Policlinico - Vittorio Emanuele”, Catania, Italy \\ ${ }^{2}$ Department of Clinical and Experimental Medicine, University of Catania, Catania, Italy
}

\section{Doi: 10.12890/2017_000540 - European Journal of Case Reports in Internal Medicine - ๑ EFIM 2016}

Received: $23 / 11 / 2016$

Accepted: $15 / 12 / 2016$

Published: $20 / 01 / 2017$

How to cite this article: Bruno CM, Pricoco GS, Bellinvia S, Maradio MD, Cantone D, Polosa R. Necrotizing panniculitis as an uncommon manifestation of acute pancreatitis. EJCRIM 2017;4: doi:10.12890/2017_000540

Conflicts of Interests: The Authors declare that there are no competing interests.

This article is licensed under a Commons Attribution Non-Commercial 4.0 License

Acknowledgements: R. Polosa and C.M. Bruno designed the report. D. Cantone collected the patient's clinical data. G.S. Pricoco, M.D. Amaradio and S. Bellinvia analyzed the data and wrote the paper.

\section{ABSTRACT}

Pancreatic panniculitis is a rare disorder affecting 2-3\% of patients with pancreatic disease. The findings are characterized by tender, erythematous, subcutaneous nodules which may undergo spontaneous ulceration with discharge of brownish and viscous material derived from colliquative necrosis of adipocytes. The lesions are usually localized in the lower limbs, although they may also extend to the buttocks and also involve the trunk, upper limbs and scalp. They can precede overt pancreatic disease in $40 \%$ of cases. The typical histological features observed in these lesions are characterized by necrotic adipocytes with absent nuclei (better known as 'ghost cells') in the context of a predominantly lobular panniculitis.

We describe the case of a 78-year-old cirrhotic woman admitted to our department with abdominal pain affecting the upper abdomen and a 3-day fever. On physical examination, multiple tender erythematous nodules, with irregular margins, were present on the pretibial regions of both lower legs, ranging in size from 0.8 to $1.5 \mathrm{~cm}$. Pancreatic amylase and lipase were elevated and abdominal computed tomography revealed acute pancreatitis with oedema, focal gland enlargement of the pancreatic tail and perivisceral inflammation. Histological examination of the lesions was consistent with a diagnosis of necrotizing granulomatous panniculitis.

\section{LEARNING POINTS}

- Identification of the aetiological factors of tender erythematous nodules is challenging.

- Careful examination and history taking is essential for correct diagnosis and proper treatment.

- Pancreatic panniculitis should be included in the differential diagnosis as it can indicate developing acute pancreatitis.

\section{KEYWORDS}

Panniculitis; acute pancreatitis; erythematous nodules; enzymatic fat necrosis; ghost adipocytes 


\section{INTRODUCTION}

Panniculitis is an inflammation of the subcutaneous adipose tissue under the skin epidermis, and usually presents with inflammatory nodules or plaques ${ }^{[1]}$. A wide variety of panniculitis subtypes exists, mainly in the geriatric population, including panniculitis related to infection, external insults, malignancy, inflammatory diseases and enzymatic destruction. This wide range of possibilities may delay the diagnosis, leading to prolonged hospitalization. Recognizing important diagnostic clues may pinpoint the correct disease, even before its onset.

\section{CASE PRESENTATION}

A 78-year-old woman was admitted to our department with abdominal pain, nausea and a 3-day fever. The pain became progressively more severe. It was affecting the epigastrium, radiating on both the left and right sides as well as posteriorly to the spine. The patient had a history of Child Pugh A cirrhosis HCV-related, multifocal hepatocellular carcinoma and gallbladder removal at the age of 60 . No other significant comorbidities were present. No history of hypertriglyceridemia, biliary colic or alcohol abuse was reported.

On the day of admission, the patient was febrile $\left(37.9^{\circ} \mathrm{C}\right)$ on physical examination, while other vital signs were normal. Heart and lung evaluations were normal. The abdominal examination revealed epigastric and mesogastric tenderness, without defensive reaction or other signs of peritoneal irritation.

The initial laboratory values showed elevated levels of serum amylase (1197 IU/I) and lipase (937 IU/I), C reactive protein (39.6 mg/dI) and liver enzymes (GPT $54 \mathrm{U} / \mathrm{I}$; GOT $32 \mathrm{U} / \mathrm{l})$. Haemoglobin was $10.1 \mathrm{~g} / \mathrm{dl}$ and mean corpuscular volume was $94.4 \mathrm{fl}$. Renal function was normal and no electrolyte abnormalities were found.

Multiple painful erythematous nodules were found on the pretibial regions of both lower legs, ranging in size from 0.8 to $1.5 \mathrm{~cm}$, with irregular margins and tender to palpation (Fig. 1).

Due to suspicion of leukocytoclastic vasculitis or lupus panniculitis, dsDNA antibodies, ANA, ENA and ANCAs tests were performed.

The following day, an excisional biopsy of a nodular lesion in the upper third of the leg was performed and revealed lobular panniculitis with foci of fat necrosis and anucleated adipocytes (Fig. 2). The diagnosis was consistent with necrotizing granulomatous panniculitis.

An abdominal CT scan showed diffuse pancreatic enlargement, with loss of normal acinar structure and peri-pancreatic fat tissue densification (Fig. 3).

A diagnosis of mild acute pancreatitis was made in accordance with the Atlanta criteria and intravenous hydration and analgesia were administered with improvement of symptoms. Pancreatic enzymes remained elevated for several days.

Supportive treatment of acute pancreatitis was maintained and the nodular lesions disappeared in 10 days, leaving residual skin scars.

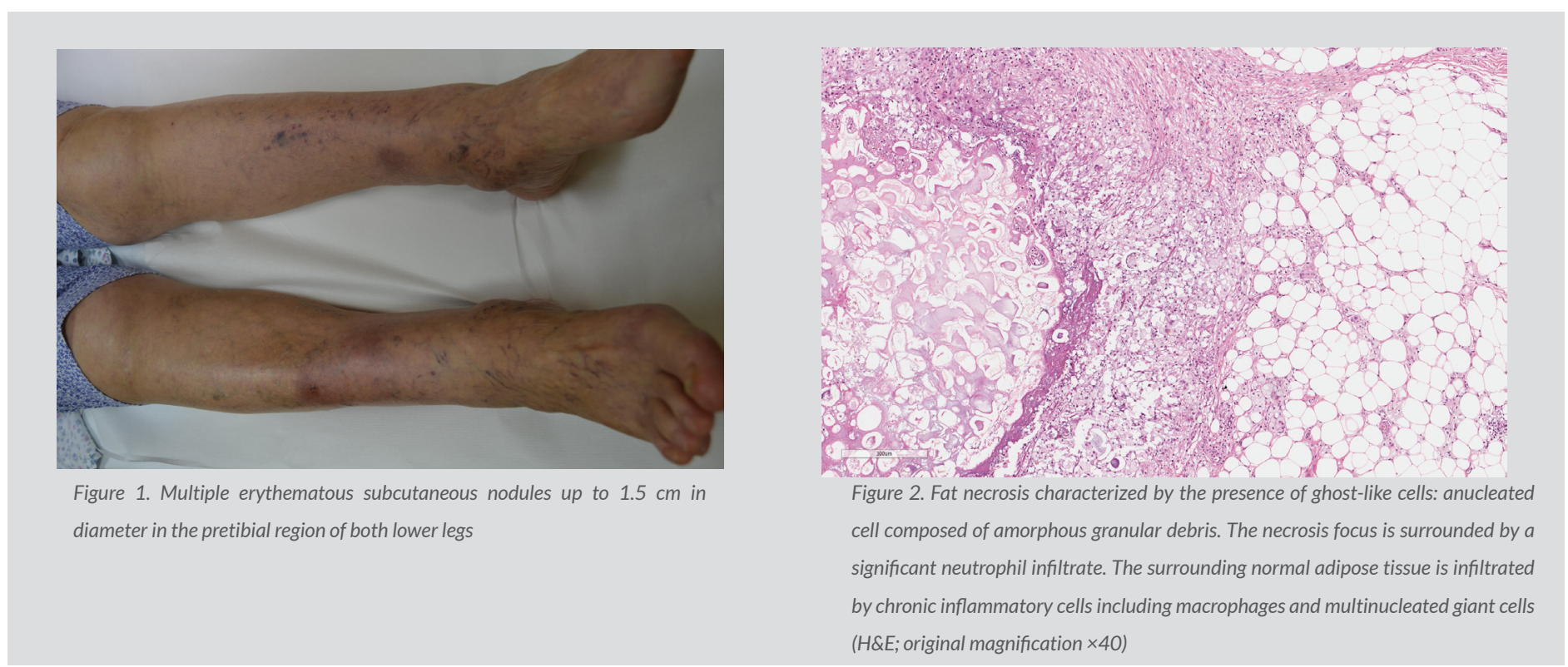




\section{DISCUSSION}

Pancreatic panniculitis is a rare disorder first described by Chiari in 1883 and affects $2-3 \%$ of all patients with pancreatic disease ${ }^{[2]}$.

About 150 case have been reported in the literature ${ }^{[3]}$. The condition is most commonly associated with alcohol-related pancreatitis, either acute or chronic, but also with pancreatic cancer, especially acinar cell carcinoma, and less frequently islet cell carcinoma ${ }^{[2]}$. Other rare causes of panniculitis are pancreatitis due to cholelithiasis, post-traumatic pancreatitis, pseudocyst and malformations such as pancreas divisum ${ }^{[2]}$. A case of pancreatic panniculitis associated with primary HIV infection and haemophagocitic syndrome has been reported ${ }^{[1]}$.

The pathophysiology is not clear: the main hypothesis is that release into the circulation of pancreatic enzymes, such as trypsin, may increase microcirculation permeability, allowing lipase and amylase to penetrate the adipose tissue causing saponification of neutral fat. The ensuing inflammation causes lobular panniculitis ${ }^{[2]}$.

The observation that not all cases of pancreatitis are associated with panniculitis together with the fact that several cases of pancreatic panniculitis with normal levels of serum pancreatic enzymes have been reported, suggest the involvement of other mechanisms. Furthermore, no 'panniculitic changes' develop when normal human fat cells are incubated in vitro with pancreatic enzymes ${ }^{[4]}$.

Zellman suggested that concomitant events such as inflammation, oedema, or altered immunity with deposition of antibody-antigen complex may damage blood vessels causing panniculitis ${ }^{[5]}$. Further studies are needed to precisely determine the pathogenesis of panniculitis.

Pancreatic panniculitis is diagnosed when pancreatic disease is associated with cutaneous lesions confirmed by typical histopathological findings in a skin biopsy specimen ${ }^{[4]}$. Typically, the patient presents with subcutaneous tender erythematous nodules, painful or asymptomatic, sometimes ulcerated or draining oily serous discharge derived from fat necrosis ${ }^{[4]}$. Generally, the nodules develop in the lower limbs, and less frequently in the arms, chest or abdomen ${ }^{[6]}$. They can appear before, during or after pancreatitis. A skin biopsy is necessary to confirm the clinical suspicion. A septal pattern has been described at a very early stage, due to enzymatic damage of endothelial septa. This allows the pancreatic enzyme to penetrate into fat lobules determining the typical lobular pattern with the formation of pathognomonic 'ghost cells $^{\prime[4]}$. These are anucleated adipocytes that, due to the resistance of the fat cell membrane to lipase, are surrounded by a poorly defined wall containing intracytoplasmic fine basophilic granulations without a nucleus. Sometimes a neutrophilic infiltrate is reported around fat necrosis, which then decreases, giving way to granulomatous lesions and, finally, to fibrosis and lipoatrophy ${ }^{[4]}$.

The treatment is that of pancreatic disease. Symptomatic treatment with non-steroidal anti-inflammatory drugs or topical steroids has not been effective. Plasmapheresis and subcutaneous octreotide used to block pancreatic enzymes production requires appraisal in further studies on larger case series ${ }^{[2]}$. Usually the nodules disappear spontaneously leaving atrophic hyperpigmentation ${ }^{[7]}$. Rare complications are disseminated fat necrosis, arthritis and synovitis, and submucosal necrosis of the bowel. Although the pancreatic panniculitis generally shows a benign course, the prognosis of patients with systemic involvement is grim ${ }^{[2]}$.

The present case report describes an uncommon cause of panniculitis due to mild acute pancreatitis. Although fever was recorded on admission, clinical, instrumental and laboratory evidence ruled out bacterial or fungal infections. Moreover, diagnostic work-up failed to detect any immune or chronic systemic inflammatory disorder. There was no evidence of a deposition cause for the skin lesions such as gouty panniculitis, calciphylaxis or hyperoxaluria.

Although cancer was considered in the differential diagnosis, the fast disappearance of the erythematous nodules after resolution of pancreatic inflammation supported our hypothesis that the panniculitis was triggered by the enzymatic destruction of subcutaneous fat tissue. 


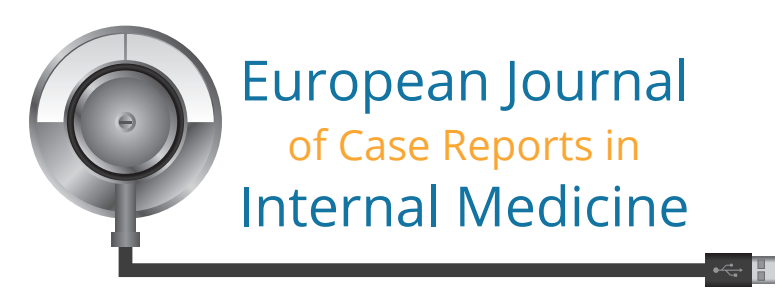

This unusual case of panniculitis shows that the identification of the underlying disease can be quite challenging. Especially in a patient presenting with clinical and anamnestic features suggesting a pancreatic disorder, the case presented clearly illustrates that the diagnostic reasoning, in addition to classic non-inflammatory and inflammatory causes of panniculitis, should also consider the association with enzymatic destruction due to release of pancreatic matter, before labelling the panniculitis origin as 'undetermined'.

\section{REFERENCES}

1. Johnson MA, Kannan DG, Balachandar TG, Jeswanth S, Rajendran S, Surendran R. Acute septal panniculitis. A cutaneous marker of a very early stage of pancreatic panniculitis indicating acute pancreatitis. JOP 2005;6:334-338

2. Lengfeld J, Kneitz H, Goebeler M, Kolb-Mäurer A. Panniculitis due to pancreatic disease. J Dtsch Dermatol Ges 2015;13:807-809.

3. Colantonio S, Beecker J. Pancreatic panniculitis. CMAJ 2012;184:E159.

4. Laureano A, Mestre T, Ricardo L, Rodrigues AM, Cardoso J. Pancreatic panniculitis - a cutaneous manifestation of acute pancreatitis. J Dermatol Case Rep 2014;8:35-37.

5. Zellman GL. Pancreatic panniculitis. J Am Acad Dermatol 1996;35(2 Pt 1):282-283.

6. Neves Z, Segura U, Valente A, Pacheco MH, Malhado J. Panniculitis - a rare manifestation of acute pancreatitis. GE Port J Gastroenterol 2015;22:117-120.

7. Lee WS, Kim MY, Kim SW, Paik CN, Kim HO, Park YM. Fatal pancreatic panniculitis associated with acute pancreatitis: a case report. J Korean Med Sci 2007;22:914-917. 